\title{
NUMERICAL IDENTITIES IN LATTICES WITH AN APPLICATION TO DIRICHLET PRODUCTS
}

\author{
EDWARD A. BENDER
}

1. Introduction. Let $\phi$ and $\psi$ be functions from the quotient lattices of a lattice $L$ to a field of characteristic zero. By analogy with the classical Dirichlet product Ward [2] has defined

$$
\begin{aligned}
(\phi+\psi)(x / y) & =\phi(x / y)+\psi(x / y), \\
(\phi \psi)(x / y) & =\sum_{x \underline{\geq} u \underline{y} y} \phi(x / u) \psi(u / y) .
\end{aligned}
$$

In order to guarantee the existence of $\phi \psi$ it is assumed that every quotient lattice of $L$ is finite. The function $\phi$ is said to be factorable if

$$
\phi(x / y) \phi(u / v)=\phi\left(\frac{x \cup u}{y \cup v}\right) \phi\left(\frac{x \cap u}{y \cap v}\right)
$$

whenever $x \supseteq y$ and $u \supseteq v$ in $L$. One of Ward's results asserts that $L$ is distributive if the factorable functions are closed under Dirichlet product. We will prove here the following stronger result.

THEOREM 3.1. The factorable functions are closed under Dirichlet product if and only if every quotient lattice of $L$ is a Boolean algebra.

This result is a consequence of a numerical identity which characterizes such lattices. Similar identities will be shown to characterize distributive and modular lattices in which all quotient lattices are finite.

Curved symbols are used for lattice relations. The number of elements in the quotient lattice $x / y$ is denoted by $|x / y| .^{1}$

2. Boolean lattices. We will call a lattice Boolean if every quotient lattice is a finite Boolean algebra. It will be shown that such lattices are characterized by the identity

$$
\left|\frac{x}{y}\right| \cdot\left|\frac{u}{v}\right|=\left|\frac{x \cup u}{y \cup v}\right| \cdot\left|\frac{x \cap u}{y \cap v}\right|
$$

whenever $x \supseteq y$ and $u \supseteq v$. Furthermore (2.1) is equivalent to the apparently weaker condition

Received by the editors November 13, 1962.

${ }^{1}$ I would like to thank Dr. R. P. Dilworth and Dr. R. A. Dean for their help in the preparation of this paper. 


$$
\left|\frac{x}{y}\right|=\left|\frac{x}{u}\right| \cdot\left|\frac{u}{y}\right|
$$

whenever $x \supseteq u \supseteq y$.

TheOREM 2.1. The following conditions are equivalent.

(i) $L$ is a Boolean lattice.

(ii) Whenever $x \supseteq y$ and $u \supseteq v$ in $L$, (2.1) holds.

(iii) Whenever $x \supseteq u \supseteq y$ in L, (2.2) holds.

Proof. If (i) holds, we may define a dimension function " $d$ " in any quotient lattice. In particular in $x \cup u / y \cap v$

$$
\begin{aligned}
\left|\frac{x}{y}\right| \cdot\left|\frac{u}{v}\right| & =2^{d(x)-d(y)+d(u)-d(v)}=2^{d(x \cup u)-d(y \cup v)+d(x \cap u)-d(y \cap v)} \\
& =\left|\frac{x \cup u}{y \cup v}\right| \cdot\left|\frac{x \cap u}{y \cap v}\right| .
\end{aligned}
$$

By specializing (2.1) to $x \supseteq u=v \supseteq y$ we deduce (2.2).

The proof that (iii) implies (i) will be divided into several parts. (a) If $N$ is the length of a maximal chain from $x$ to $y$, then $|x / y|$ $=2^{N}$.

PROOF. Let $x=u_{1}>u_{2}>\cdots>u_{N+1}=y$, where $>$ indicates covering. Clearly

$$
\left|\frac{x}{y}\right|=\left|\frac{u_{1}}{u_{2}}\right| \cdot\left|\frac{u_{2}}{u_{3}}\right| \cdot \ldots \cdot\left|\frac{u_{N}}{u_{N+1}}\right|=2^{N} .
$$

(b) Every element in $x / y$ is the join of elements covering $y$.

Proof. Let $u \neq y$ be join irreducible in $x / y$ and let $v<u$. Then $|u / y|=1+|v / y|$, but $|u / y|=2|v / y|$. Hence $|v / y|=1$ and thus $v=y$.

To complete the theorem we proceed by induction on $|x / y|$. Assume that the theorem holds for $x / y$ if $|x / y|<|u / v|=2^{N}$. Choose $w<u$. There exists $p>v$ and $p \notin w / v$ since $u$ is the join of elements covering $v$.

(c) Every element of $u / v$ belongs to one of $u / p$ and $w / v$.

Proof. $|w / v|+|u / p|=2^{N-1}+2^{N-1}=|u / v|$. If $z \in w / v$ and $z \in u / p$, then $w \supseteq z \supseteq p$ contradicting $p \notin w / v$.

(d) $u / v$ is a Boolean algebra.

Proof. It suffices to show that each element is the unique join of points (elements covering $v$ ). By the induction hypothesis $w / v$ has $N-1$ points. Thus $u / v$ has $N$ points by (c). The number of subsets of a set of $N$ elements is $2^{N}$. Since $|u / v|=2^{N}$ and every element of $u / v$ is a join of points, the result follows. 
3. An application to Dirichlet product. This section establishes the result stated in the introduction.

Theorem 3.1. The factorable functions are closed under Dirichlet product if and only if $L$ is a Boolean lattice.

Proof. Assume the factorable functions are closed under Dirichlet product. Since $\zeta=1$ is factorable and $\zeta^{2}(x / y)=|x / y|$, we conclude by (1.2) and Theorem 2.1 that $L$ is a Boolean lattice.

Assume that $L$ is a Boolean lattice and let $\phi$ and $\psi$ be factorable functions, then we must show that $\theta=\phi \psi$ is factorable. By (1.1) and (1.2)

$$
\begin{aligned}
& \theta(x / y) \theta(u / v)
\end{aligned}
$$

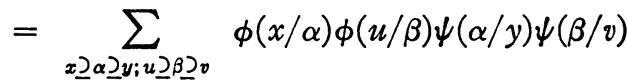

$$
\begin{aligned}
& =\sum_{x \supseteq \alpha \supseteq y_{i} u \supset \beta \supset v} \phi\left(\frac{x \cup u}{\alpha \cup \beta}\right) \phi\left(\frac{x \cap u}{\alpha \cap \beta}\right) \psi\left(\frac{\alpha \cup \beta}{y \cup v}\right) \psi\left(\frac{\alpha \cap \beta}{y \cap v}\right)
\end{aligned}
$$

and

$$
\begin{aligned}
& \theta\left(\frac{x \cup u}{y \cup v}\right) \theta\left(\frac{x \cap u}{y \cap v}\right)
\end{aligned}
$$

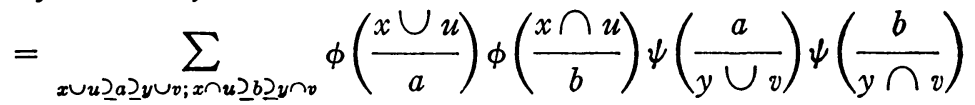

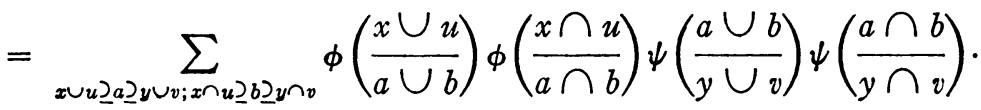

By Theorem 2.1 (3.1) and (3.2) have the same number of terms so we need only exhibit a mapping $f(\alpha, \beta)=(a, b)$ such that when $x \supseteq \alpha \supseteq y$ and $u \supseteq \beta \supseteq v f$ is one-to-one, $\alpha \cup \beta=a \cup b, \alpha \cap \beta=a \cap b$, $x \cup u \supseteq a \supseteq y \cup v, x \cap u \supseteq b \supseteq y \cap v$. Let $q=y^{\prime} \cap x \cap v^{\prime} \cap u$ (' denotes complement relative to $x \cup u / y \cap v), p=y^{\prime} \cap v \cap x, r=y^{\prime} \cap u^{\prime} \cap x, d=\alpha \cap q$, $e=\beta \cap q, s=(\alpha \cup \beta) \cap q^{\prime}, t=(\alpha \cap \beta) \cap q^{\prime}, a=d \cup s$, and $b=e \cup t$. Since $d=a \cap q, s=a \cap q^{\prime}, t=b \cap q^{\prime}$,

$$
\begin{aligned}
y \cup(r \cap s) \cup(p & \cap t) \cup d \\
& =y \cup[r \cap(\alpha \cup \beta)] \cup[p \cap \alpha \cap \beta] \cup[\alpha \cap q] \\
& =y \cup(r \cap \alpha) \cup(r \cap \beta) \cup(p \cap \alpha) \cup(q \cap \alpha) \\
& =y \cup[\alpha \cap(r \cup p \cup q)]=y \cup\left(\alpha \cap x \cap y^{\prime}\right)=\alpha,
\end{aligned}
$$

and similarly for $\beta$, the mapping is one-to-one. Also $a \cup b=d \cup e \cup s$ $=\alpha \cup \beta$ and $a \cap b=(d \cup s) \cap(e \cup t)=(d \cap e) \cup t=\alpha \cap \beta$. Clearly 
$x \cup u \supseteq a \supseteq s=(\alpha \cup \beta) \cap q^{\prime} \supseteq(y \cup v) \cap(y \cup v)=y \cup v$,
$y \cap v \subseteq b=(\beta \cap q) \cup\left(\alpha \cap \beta \cap q^{\prime}\right) \subseteq(u \cap q) \cup(x \cap u)=x \cap u$.

4. Other conditions. By imposing restrictions on

$$
\left|\frac{x}{y}\right| \cdot\left|\frac{u}{v}\right|=\left|\frac{x \cup u}{y \cup v}\right| \cdot\left|\frac{x \cap u}{y \cap v}\right|
$$

we can obtain various other identities. The most interesting appear to be the conditions $v=u, x=u$ taken in conjunction with $x=y \cup v$ or by themselves.

A. $v=u$ and $x=y \cup v$ give the well-known result

THEOREM 4.1. The lattice $L$ is modular if and only if for all $x, y \in L$

$$
\left|\frac{x \cup y}{x}\right|=\left|\frac{y}{x \cap y}\right| \text {. }
$$

B. $x=u=y \cup v$ gives the identity

$$
\left|\frac{x \cup y}{x \cap y}\right|=\left|\frac{x \cup y}{x}\right| \cdot\left|\frac{x \cup y}{y}\right|
$$

which has the dual

$$
\left|\frac{x \cup y}{x \cap y}\right|=\left|\frac{x}{x \cap y}\right| \cdot\left|\frac{y}{x \cap y}\right| \text {. }
$$

THEOREM 4.2. The following conditions are equivalent.

(i) The lattice $L$ is distributive.

(ii) Given any $x, y \in L,(4.1)$ holds.

(iii) Given any $x, y \in L,(4.2)$ holds.

Proof. By duality it is sufficient to show that (i) and (iii) are equivalent.

If $L$ is distributive we will show that

$$
\frac{x \cup y}{x \cap y} \cong \frac{x}{x \cap y} \times \frac{y}{x \cap y}
$$

If $u \in L$ let $f(u)=(x \cap u, y \cap u)$. Whenever $u \in x \cup y / x \cap y,(x \cap u)$ $\cup(y \cap u)=u \cap(x \cup y)=u$; therefore, the mapping is one-to-one. Let

$$
(w, v) \in \frac{x}{x \cap y} \times \frac{y}{x \cap y},
$$

then by setting $u=w \cup v$ we get $u \in x \cup y / x \cap y, x \cap u=w, y \cap u=v$. 
Thus $f$ is onto. Finally, for $u, v \in x \cup y / x \cap y, f(u) \cap f(v)=f(u \cap v)$ and by distributivity $f(u) \cup f(v)=f(u \cup v)$.

If (iii) holds $L$ must be modular for otherwise it contains a sublattice like that shown in Figure 1 and

$$
\left|\frac{x}{z}\right| \cdot\left|\frac{w}{z}\right|=\left|\frac{u}{z}\right|=\left|\frac{y}{z}\right| \cdot\left|\frac{w}{z}\right|<\left|\frac{x}{z}\right| \cdot\left|\frac{w}{z}\right| .
$$

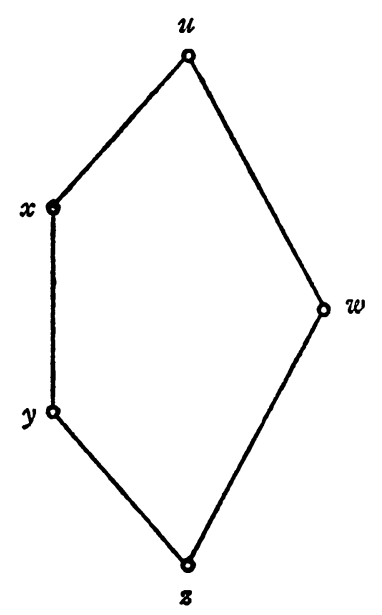

Figure 1

If $L$ is not distributive it contains $u>w, x, y>z$ (Ward [2]) and $5 \leqq|u / z|=|x / z| \cdot|y / z|=4$.

Actually a somewhat stronger version of the theorem can be proved; namely, (iii) may be replaced by the weaker condition (iii)': Given any $x, y \in L$ either (4.1) or (4.2) holds.

C. $x=u$ leads to the identity

$$
\left|\frac{v}{x \cup y}\right| \cdot\left|\frac{v}{x \cap y}\right|=\left|\frac{v}{x}\right| \cdot\left|\frac{v}{y}\right|
$$

whenever $v \supseteq x \cup y$, which has the dual

$$
\left|\frac{x \cap y}{v}\right| \cdot\left|\frac{x \cup y}{v}\right|=\left|\frac{x}{v}\right| \cdot\left|\frac{y}{v}\right| .
$$

Let $S$ be the set of all vectors of dimension $N$ (possibly infinite) over the integers with at most a finite number of nonzero components and satisfying inequalities of the form $a_{\alpha}<x_{\alpha}<b_{\alpha}$, where $-\infty \leqq a_{\alpha} \leqq 0$ and $0 \leqq b_{\alpha} \leqq+\infty$. Order $S$ by writing $x \leqq y$ if and only if $x_{\alpha} \leqq y_{\alpha}$ for 
all components of $x$ and $y$. By this means we make $S$ into a lattice. We state without proof

THEOREM 4.3. The lattice $L$ has the above form if and only if for all $x, y, v \in L$ (4.3) and (4.4) hold.

It is clear that $L$ is a Boolean lattice if and only if $L$ is of the above type with the additional restriction that each component can assume only two values.

D. $u=v$ is easily seen to be equivalent to the condition that $L$ is Boolean by assuming $x \supseteq u \supseteq y$ and applying Theorem 2.1.

\section{REFERENCES}

1. G. Birkhoff, Lattice theory, rev. ed., Amer. Math. Soc. Colloq. Publ. Vol. 25, Amer. Math. Soc., Providence, R. I., 1948.

2. M. Ward, The algebra of lattice functions, Duke Math. J. 5 (1939), 357-371.

California Institute of Technology 